\title{
Les normes comme
}

\section{démarche collective}

\begin{abstract}
Le processus de normalisation est intrinsèquement une démarche collective qui illustre parfaitement le concept de « stratégie collective » proposé par Astley et Fombrun (1983). Toutefois, cette théorisation constitue-t-elle un modèle opérant dans le champ du marketing stratégique? Une étude empirique menée auprès de 150 entreprises adhérentes de I'AFNOR (Association française de normalisation) permet de vérifier la validité empirique de ces formes stratégiques, de comprendre comment ces stratégies peuvent être mises en œuvre et de caractériser les entreprises qui les adoptent.
\end{abstract}

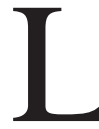
e concept de « stratégie collective », tel que proposé par Astley et Fombrun (1983) dans l'approche de l' « écologie organisationnelle », offre une perspective innovante pour l'analyse stratégique (Le Roy, 2003 ; Yami, 2003). Selon cette approche, l'action stratégique proactive se situe au niveau de la définition même des conditions de l'environnement: il s'agit de construire un « environnement négocié » (Cyert et March, 1963) pour se protéger des menaces extérieures, cet environnement se réalisant au travers d'une « interaction appropriée » entre les acteurs du marché. Le fondement conceptuel (la proactivité) et l'objectif des stratégies collectives (l'environnement « négocié ») sont explicités, et les auteurs présentent une typologie pour représenter les formes que peuvent adopter ces stratégies (Astley et Fombrun, 1983). Au-delà des aspects théoriques qui ont donné lieu à une solide littérature (Astley et Fombrun, 1983; Astley, 1984; Bresser et Harl, 1986; Bresser, 1988), il existe peu de recherches qui tentent de vérifier la validité empirique de ces formes stratégiques, de comprendre comment ces stratégies peuvent être mises en œuvre et d'essayer de caractériser les entreprises qui les adoptent. 
Dans cette perspective, nous considérons le cas des normes qui paraissent constituer un modèle archétypal des stratégies collectives. Les normes peuvent être entendues comme instrument ou comme modalité de stratégies collectives, selon que l'on considère la norme comme une règle (résultat $\mathrm{du}$ processus) ou comme processus d'organisation lui-même. Cette double dimension en fait un instrument d'analyse privilégié du collectif, de son organisation et des règles d'organisation.

Ainsi, les normes de droit, auxquelles nous limitons notre étude, constituent un cadre d'analyse pertinent des stratégies collectives pour deux raisons: parce que leur mode de définition est nécessairement consensuel et qu'elles rendent possible la proactivité. En premier lieu, le mode de définition des normes de droit est une procédure institutionnelle fondée sur la négociation et la concertation entre tous les acteurs du marché. Le processus de création des normes est donc par nature une stratégie collective. Deuxièmement, le processus de création des normes constitue une stratégie proactive en ce que les normes définissent les conditions du jeu concurrentiel. En effet, les quatre fonctions principales des normes-information, réduction de la variété, compatibilité et qualité-structurent les conditions du marché. Les normes fixent certaines caractéristiques des produits et autorisent ou interdisent ainsi l'accès des produits à un marché, elles préconisent des méthodes pour les concevoir et les réaliser, indiquent les moyens de l'interopérabilité ou de la compatibilité, exigent des niveaux de performance à atteindre pour obtenir des signes de qualité. Agir sur la définition des normes, lors des travaux normatifs, voire adopter certaines normes pour favoriser leur diffusion peuvent exprimer donc l'intentionnalité proactive d'agir sur les conditions même de l'environnement.

Si les propriétés structurantes des normes paraissent indéniables, le caractère instrumental des normes - la possibilité de s'en servir dans une optique utilitariste - pose question. Les économistes de la standardisation ont mis en évidence les processus de structuration parfois irrationnels qui conduisent à la domination d'un standard sur l'autre, sans motif rationnel, dans les technologies de réseau (Arthur, 1987,1989; Cowan et Foray, 1998; David, 1987 ; David et Greenstein, 1990; Foray 1990, 1993, 1996). Ces effets structurants font également l'objet des analyses des psychosociologues et des institutionnalistes qui montrent comment les normes qui sont apprises, puis intériorisées structurent les comportements des acteurs au sein des organisations et l'isomorphisme des organisations ellesmêmes (Di Maggio et Powell, 1983). Ces optiques soulignent le caractère structurant des normes sur les perceptions et les comportements. La position qui est adoptée ici prend en considération ces effets structurants, mais privilégie un ancrage dynamique et observe le décideur, sa perception des effets des normes et sa conception de cet instrument.

Précisément, nous considérons que l'emploi des normes, comme modalité de stratégie collective, renvoie à une double dimension perceptuelle et comportementale. Il s'agit de percevoir les propriétés proactives des normes et de les utiliser pour modifier les conditions environnementales. L'enquête propose d'évaluer empiriquement la prégnance de cette stratégie. Elle présente deux phases méthodologiques: une phase qualitative sur base d'entretiens approfon- 
dis est réalisée auprès de quatorze responsables centraux de normalisation des grands groupes industriels français. Cette phase permet de préciser les différents niveaux stratégiques auxquels les normes peuvent être associées: le niveau interorganisationnel (marché), le niveau corporate (entreprise), le niveau business (produits-marchés). Ces niveaux sont présentés par Astley et Fombrun, dans leur article fondateur de 1983 : le niveau interorganisationnel est décrit comme celui qui permet d'identifier les stratégies collectives. L'enquête qualitative fait apparaître que les normes sont davantage utilisées par les entreprises de grande taille, leaders sur leur marché, adoptant un profil innovant, appartenant aux secteurs technologiques et ayant structuré la gestion des normes. Les hypothèses testées posent que la perception des propriétés stratégiques des normes et ainsi que le comportement vis-à-vis des normes varient en fonction de ces caractéristiques. L'enquête, effectuée auprès de cent cinquante deux entreprises adhérentes de l'AFNOR, interroge deux responsables par entreprise, le responsable des normes et le responsable marketing. Ainsi, nous proposons une méthodologie permettant la mise en évidence d'un mode de gestion effectif des normes en ce qu'il doit se révéler dans les perceptions des propriétés stratégiques des normes par les deux responsables, dans les comportements vis-à-vis des normes, dans une coordination entre eux et dans le comportement sur le marché.

Les résultats de notre recherche mettent en évidence que les propriétés des normes à structurer un marché sont effectivement perçues et que les intentions stratégiques pour l'emploi de ces propriétés peuvent être coopératives, notamment pour protéger un marché et développer un marché naissant, ou agressives pour dominer un marché, accélérer la diffusion de sa technologie ou ralentir celle d'un concurrent. Ces dimensions coopératives et concurrentielles coexistent et le cadre des normes qui apparaît est un terrain privilégié pour donner corps au concept de coopétition. L'emploi de ces propriétés n'est toutefois pas le fait de toutes les entreprises. Une typologie fait apparaître une gestion proactive des normes de la part de grandes entreprises, structurées, leaders sur leurs marchés, innovatrices et appartenant à un univers technologique. Nous discutons alors des dimensions réactive et proactive, concurrentielle ou coopérative qu'offrent les situations décisionnelles spécifiques au moment des travaux normatifs, puis dans la mise en conformité.

\section{I. - STRATÉGIES COLLECTIVES ET NORMES}

\section{Stratégies collectives, une perspective proactive et interorganisationnelle}

Astley et Fombrun décrivent quatre idéaux types selon le mode de coordination (direct ou indirect) et la forme de l'interdépendance (liant des entreprises de même nature ou différentes) : les stratégies confédérées, conjuguées, agglomérées ou organiques. Pour notre part, nous considérons que les normes s'apparenteraient aux formes d'association de type indirect, concernant les types d'interdépendance horizontale et verticale, soit les formes « agglomérée » (avec coordination centralisée) ou « organiques » (relations résiliaires informelles). Cependant, dans notre réflexion, nous ne nous attachons pas à caractériser ces formes. Du 
modèle des stratégies collectives, nous souhaitons mettre en évidence deux dimensions: la proactivité et le niveau d'analyse interorganisationnel.

\section{Proactivité des stratégies collectives}

La proactivité est le fondement même des stratégies collectives. À l'origine de ce construit théorique, se pose le constat de l'impuissance des organisations individuellement à jouer un rôle proactif sur l'environnement: « [...] à un niveau macroéconomique, historique, politique, économique, les facteurs sociaux déterminent le destin de populations entières d'organisations, de sorte que les actions d'organisations seules comptent pour peu dans le cours des événements sur le long terme $»^{1}$ (Astley et Fombrun, 1983, p. 577). Pour réaliser cette démonstration, Astley et Fombrum s'appuient sur des auteurs tels que (Hannan et Freeman, 1977) ou Aldrich (1979) qui considèrent que l'environnement détermine l'activité organisationnelle et que la liberté de «choix stratégique » de Child (1972) n'est qu'illusoire. Les auteurs admettent avec Pfeffer et Salancik (1978) que le management peut se prévaloir d'un rôle symbolique, mais que ce rôle est largement déconnecté de conséquences en termes d'efficacité ou de survie. Finalement, les managers réagissent aux contraintes extérieures en percevant et en traitant l'information pour identifier les tendances qui ne sont pas sous le contrôle de l'organisation (Andrew, 1971; Bourgeois, 1980 ; Hofer et Schendel, 1978), et l'action stratégique consiste à faire coller les capacités organisationnelles aux exigences environnementales (Astley et Fombrun, 1983).

Il existe pourtant une façon d'agir sur l'environnement: Pfeffer et Salancik (1978) identifient des façons de manager des sources d'interdépendances à l'environnement et les tenants du « stakeholder management » (Ansoff, 1965; Emshoff et Freeman, 1981; Mason et Mitroff, 1981) incitent à identifier des groupes d'intérêt spécifiques qui sont critiques pour la vie de l'organisation. Ainsi, à travers une interaction appropriée avec ces groupes d'intérêt, le management peut légitimer et stabiliser son existence, en se protégeant contre les menaces extérieures par la construction d'un environnement négocié (Cyert et March, 1963). Le choix stratégique comme processus proactif existe dans une gestion collective de l'interorganisationnel.

\section{Normes, proactivité et niveau interorganisationnel}

Les théoriciens de la planification sociale comme Trist (1979), Ackoff (1974), Michaël (1973), Vickers (1965), et Schon (1971) soulignent que la turbulence de l'environnement moderne tient à ce que les éléments sont interconnectés et interdépendants et que l'action, au niveau collectif, permet de stabiliser cette turbulence en évitant le chaos produit par des actions individuelles contradictoires. Ackoff (1974) propose le terme de «planning interactif », Michaël (1973) celui d' « apprentissage social de la modernité » (future-responsive social learning), Emery et Trist (1973) soutiennent l'adoption de l'approche de l'écologie sociale. Ces approches préconi-

1. "[...] at a macro level, historical, political, economic, and social factors determine the fate of whole populations of organizations, so that the actions of single organizations count for little in the long run course of events." 
sent la construction d'un environnement social plus contrôlé, plus stable et prévisible que l'environnement naturel.

La construction de cet environnement suppose la mise en évidence d'un troisième niveau de décisions stratégiques, le niveau interorganisationnel, qui complète les niveaux business (avantage concurrentiel) et corporate (choix des activités), largement explorés dans la littérature en management stratégique. Il s'agit de coordonner les activités en développant des interconnections entre les entreprises et avec leur instance de régulation (Aldrich, 1979; Astley et Fombrun, 1983; Pennings, 1981). Les normes, précisément, réalisent des interconnections entre les entreprises parce qu'elles constituent le langage, la référence commune des échanges. L'Association française de normalisation, organisme institutionnel français chargé de définir les normes est l'instance de régulation.

\section{Proactivité des normes}

Les normes sont extrêmement nombreuses, diverses et variées et adopter un point de vue multisectoriel n'est pas aisé. Ainsi, nous limitons notre étude aux normes de droit (de jure - mode de définition institutionnel) qui s'opposent aux normes de fait (de facto - émergence spontanée sur le marché). Ces normes sont publiques et en ce sens ouvertes (les organismes de normalisation nationaux et internationaux publient et vendent les normes au public) à l'inverse des standards privés qui peuvent être ouverts (par l'achat de licences) ou fermés. La dimension proactive des normes tient à leur définition même. La norme technique de droit est « un document de référence destiné à la résolution de problèmes techniques et commerciaux qui se posent de façon répétée entre les partenaires économiques et commerciaux »(AFNOR, 1994). Elle se présente sous la forme d'un document écrit, approuvé par un organisme qualifié et reconnu, cet organisme pouvant être régional, national ou international. Elle est établie selon une méthode faisant appel à toutes les parties concernées, dans l'intérêt de tous. Enfin, la norme n'est pas obligatoire. La proactivité se situe dans le caractère structurant des normes sur les échanges interorganisationnels.

\section{Normes et niveau interorganisationnel}

La perspective des stratégies collectives se situe à un niveau interorganisationnel et suppose un processus collectif permettant de construire « un environnement négocié relativement stable par les interconnexions entre entreprises concurrentes et partenaires ainsi que les autres acteurs du marché, au moyen de la circulation d'informations permettant de réagir face aux turbulences de l'environnement et de rendre le comportement des autres plus prévisible » (Astley et Fombrun, 1983). Or, les normes sont un contenu d'information désignant les savoirfaire techniques du moment permettant d'adopter un langage commun et de diffuser de nouveaux concepts entre les différents acteurs du marché. Aux producteurs, elle apporte des références d'appréciation de leur marché, des standards permettant de fixer des gammes économiques et de dégager des économies d'échelle. Par la définition des conditions de compatibilité, elle leur permet de s'insérer de façon cohérente dans le système de production. Aux clients, consommateurs ou utilisateurs, elle apporte une information précise, condition de la concurrence. Elle garantit également que les produits sont normalement utilisables, 
qu'ils répondent aux conditions de sécurité, d'hygiène et de protection de l'environnement, qu'ils sont compatibles avec les autres produits auxquels ils peuvent être couplés et enfin, qu'ils ont des performances en termes de service conformes aux exigences actuelles du marché.

La norme structure les règles du jeu concurrentiel, intervenir sur la définition même est clairement l'expression d'une stratégie proactive. Le processus de création des normes de droit rassemble les différents acteurs au sein d'instances de régulation avec la mission d'aboutir à un socle commun de connaissances. Les relations qui se nouent alors au sein de comités techniques entre concurrents, offreurs et représentants des différentes instances professionnelles (syndicats professionnels, représentants des consommateurs, délégués de la protection de l'environnement) sont intéressantes à observer lorsque l'on adopte une approche dynamique de la concurrence (Le Roy, 2003) et que l'on considère les relations comme objet d'étude privilégié. Les négociations sont le lieu privilégié d'ententes qui sont indispensables pour aboutir à une position commune. Ce véritable « jeu de go » place notamment les concurrents dans des situations nécessairement coopératives. Nous observons que tant dans la mise en conformité des normes que dans la participation aux travaux normatifs, la norme constitue une grille d'analyse pertinente de la complexité des relations: elle autorise une palette très nuancée de comportements « coopétitifs » (Brandenburger et Nalebuff, 1996). Il s'agit de s'entendre sur certains éléments, pour définir des règles du jeu communes, avant que la compétition ne reprenne. En ce sens, la norme est le lieu du statu quo, le no man's land. Mais la norme permet aussi des ententes circonstanciées dont le but est la domination de la technologie choisie sur le marché. La dimension collective de l'instrument n'est pas incompatible, loin s'en faut, d'intentions individuelles. L'enquête réalisée met en évidence la complexité des relations concurrentielles entre coopération et concurrence, connivence et agressivité.

\section{II. - LA PERCEPTION DES NORMES COMME MODALITÉ DE STRATÉGIE COLLECTIVE}

L'enquête se déroule en deux phases. Une première étude qualitative est réalisée auprès de quatorze responsables centraux de normalisation (responsables des grands groupes industriels se rassemblant au sein de l'AFNOR pour définir la position française). Les entretiens en profondeur permettent de comprendre comment la norme peut être perçue, en tant que contrainte ou instrument, individuel ou collectif, et de définir des hypothèses descriptives des entreprises selon leur perception et l'emploi des propriétés stratégiques des normes. L'enquête quantitative, réalisée en 1994, a pour but de mesurer plus précisément des modes de gestion des normes et de vérifier l'hypothèse principale selon laquelle la perception des propriétés stratégiques et le comportement vis-à-vis des normes sont liées aux caractéristiques de l'entreprise.

\section{Éléments méthodologiques}

Les options choisies lors de la phase quantitative ont tenté de résoudre les deux principales difficultés inhérentes au sujet apparues lors de l'enquête qualitative: la grande 
diversité des normes et l'identification de la personne à interroger.

\section{Échantillonnage, choix des personnes à interroger et mode d'administration}

Notre cible est constituée par des entreprises suffisamment concernées par les normes pour les concevoir comme contrainte ou instrument. L'adhésion à un organisme de normalisation semble constituer un indicateur satisfaisant, elle suppose que l'entreprise achète des normes, peut être informée des projets en cours et participer aux travaux normatifs. La population mère est donc constituée des 4000 établissements adhérents de l'AFNOR. Nous adoptons la méthode des quotas pour représenter les différents secteurs concernés (industrie, service, commerce) mais notre approche demeure un échantillon de convenance car, plutôt que la représentativité, nous faisons le choix d'une analyse approfondie par entreprise. Pour des raisons, budgétaires, le choix d'interroger deux personnes par entreprise conduit effectivement à limiter le nombre des entreprises de trois cents envisagé à cent cinquante.

Seul le responsable des normes est en mesure de décrire le fonctionnement de l'activité liée aux normes dans l'entreprise; seul le responsable des marchés est capable de répondre de la prise en compte ou non des normes sur les marchés; enfin, il semble intéressant de vérifier que les propriétés des normes sont effectivement perçues et utilisées par l'un et l'autre des responsables. Les données perceptuelles concernant les propriétés stratégiques des normes sont posées aux deux responsables et les données comportementales concernant la gestion des normes ou la gestion commerciale concer- nent l'un ou l'autre des responsables. L'enquête est menée par téléphone.

\section{Le traitement des données}

Nous avons procédé aux analyses suivantes: analyses factorielles des correspondances multiples du comportement vis-à-vis des normes, du comportement de coopération entre responsables, des perceptions, de la synthèse de ces éléments. Ces analyses factorielles ont été enrichies d'une recherche de typologie au moyen de la méthode de classification et nous avons proposé une typologie des comportements vis-à-vis des normes, des comportements de coopération et une typologie selon les perceptions. Le recueil de ces dernières variables sous la forme d'échelles d'intervalle de type Lickert a permis de soumettre notre échelle de mesure des perceptions au test de fiabilité au moyen de l'alpha de Cronbach. Nous avons ensuite mené une analyse systématique des fréquences croisées entre variables descriptives (caractéristiques d'entreprise et organisation des normes) et chacune des composantes pour lesquelles nous avions formulé des hypothèses dont nous avons testé la validité au moyen du Chi deux.

Le construit: "perception des propriétés stratégiques des normes »

Conformément au modèle d'Astley et Fombrun, nous distinguons trois niveaux: interorganisationnel, corporate ou business. Nous définissons ces niveaux en fonction des objectifs stratégiques liés à l'emploi des normes, selon qu'ils concernent le marché lui-même, l'entreprise ou les produitsmarchés. 
Le niveau interorganisationnel

Il suppose la perception des normes comme un moyen collectif d'organiser un marché, avec le but de favoriser le marché luimême et non sa propre part de marché. Deux effets sont identifiés dans l'enquête qualitative: l'effet protection et l'effet développement.

En premier lieu, considérons l'effet protection. Les normes sont rapidement associées à l'opportunité d'ériger des barrières à l'entrée. Porter (1985) cite explicitement les normes, barrière à l'entrée contre les nouveaux entrants et Einsenhardt et Schoonhoven (1996) observent que les stratégies d'alliances entre concurrents directs sont souvent utilisées pour dissuader un nouveau concurrent d'entrer sur le marché. La stratégie mise en œuvre pour utiliser les normes dans ce sens suppose la coopération des concurrents internes contre ceux que l'on cherche à maintenir hors du champ concurrentiel. L'enquête qualitative nous permet de définir trois composantes pour représenter cette dimension. Il s'agit : de se protéger de la concurrence, de constituer un frein sur un marché, d'empêcher la concurrence déloyale.

Une autre dimension coopérative apparaît nettement dans l'enquête qualitative: le souhait de favoriser le développement d'un marché naissant. Les chercheurs concernant les stratégies collectives soulignent que la turbulence de l'environnement (Carney, 1987 ; Burgers et al., 1993), l'instabilité du secteur (Poissonnier, 2004), sont des facteurs favorables à l'occurrence des stratégies collectives. Pour donner les composantes d'une stratégie collective destinée à développer les marchés naissants, nous nous inspirons de l'observation de Porter
(1985) qui remarque que la réussite de la firme dans le secteur naissant dépend pour partie des autres firmes du secteur. Porter observe que le problème primordial du secteur consiste à favoriser le remplacement des produits et à attirer les premiers acheteurs et qu'au cours de cette phase la firme a en général intérêt à encourager la normalisation, à exercer une surveillance sur les produits de qualité inférieure aux normes et sur les producteurs irresponsables, et à opposer une attitude cohérente aux fournisseurs, aux clients, à l'État et à la communauté financière. La dimension « développement » est donc décrite par les composantes: convaincre de nouveaux acheteurs, assainir le marché, garantir une qualité minimale au marché. Protection du marché ou développement du marché sont associés au niveau interorganisationnel. La logique est inverse dans la recherche d'un avantage concurrentiel aux niveaux corporate et business qui donne la priorité à la part de marché.

\section{Le niveau corporate}

La perspective adoptée ici n'est plus coopérative mais individuelle, et nous définissons cette dimension de façon à d'identifier une certaine agressivité concurrentielle, dont la presse se fait l'écho au travers des batailles normatives. Ces batailles sont souvent virulentes dans les secteurs de l'innovation technologique et font apparaître des enjeux de risque et de temps. Le terme de «pari technologique » convient dans un domaine où il importe de rentabiliser les efforts de recherche et développement. La participation aux travaux normatifs permet alors une proactivité qui est favorable à l'entreprise et dans cette optique, les normes sont 
destinées à entériner une avance ou à retarder les concurrents. Les stratégies de l'innovation, la typologie de Miles et Snow (1978) et le marketing technologique nous permettent d'opérationnaliser la norme en tant qu'arme concurrentielle. Elle est décrite par ses capacités à accélérer la diffusion d'une technologie, ralentir la diffusion d'une technologie concurrente, gagner une avance sur les concurrents.

La mise en conformité aux normes permet également de se différencier des concurrents. Le timing est alors important, car être le seul ou le premier à satisfaire les exigences d'une norme apporte un avantage indiscutable sur les concurrents. Les décideurs sont interrogés sur l'importance accordée au fait d'être le premier ou le seul à satisfaire aux exigences de la norme. Le troisième niveau n'est pas directement formulé par une approche concurrentielle. Il s'agit de réaliser les activités de la meilleure façon possible sur un marché donné.

\section{Le niveau business}

$\mathrm{Au}$ niveau du produit-marché, l'approche portérienne nous semble présenter des avantages. Une approche fondée sur les ressources demanderait que l'on soit attentif à leur caractère idiosyncrasique et non imitable; elle permettrait également d'évaluer l'apprentissage organisationnel que constitue l'adoption d'une norme en termes de compétence. La position portérienne présente l'avantage de limiter la portée à la position sur le marché. Les éléments significatifs à ce titre sont la répercussion effective sur le prix des économies réalisées au niveau de la stratégie de coût et l'acceptation par le client de payer un supplément de prix pour le plus dans la stratégie de différenciation. La norme sert une stratégie de leadership de coûts lorsqu'elle permet de réaliser des économies d'échelle, de vendre un volume plus important, de réduire les variétés. Elle sert une stratégie de différenciation lorsqu'elle permet de garantir la qualité des composants, d'offrir un plus pour lequel le client sera prêt à payer un supplément de prix, de différencier le produit aux yeux de l'acheteur.

Les normes peuvent être perçues comme une contrainte ou un instrument à ces trois niveaux de perspective. Ceux-ci permettent de présenter une échelle de mesure des propriétés stratégiques des normes puis de qualifier plus précisément le type de management proactif des normes.

\section{III. - RÉSULTATS}

Les résultats de l'enquête permettent d'évaluer la fiabilité de l'échelle de mesure concernant la perception des propriétés stratégiques des normes, de présenter synthétiquement les typologies de perception et de comportement, de tester les hypothèses principales, puis de préciser les enseignements principaux liés aux normes comme modalité de stratégies collective.

\section{Une échelle de mesure}

La fiabilité de l'échelle de mesure de la perception des propriétés stratégiques des normes, décomposée selon les dimensions interorganisationnelle, corporate ou business et soumise aux deux responsables des normes et du marketing (304 personnes) est évaluée à 0,79 , ce qui est considéré comme satisfaisant compte tenu des règles empiriques en vigueur. 


\begin{tabular}{|c|c|c|c|c|}
\hline \multicolumn{4}{|c|}{$\begin{array}{c}\text { Tableau } 1 \\
\text { ÉCHELLE DE MESURE. PERCEPTION DES PROPRIÉTÉS STRATÉGIQUES } \\
\text { DES NORMES }\end{array}$} & \\
\hline Niveau & Dimensions & Composantes & $\begin{array}{l}\text { Valeur de l'alpha } \\
\text { de Cronbach }\end{array}$ & \\
\hline $\begin{array}{l}\text { Interorganisa- } \\
\text { tionnel }\end{array}$ & $\begin{array}{l}\text { Protection } \\
\text { Développement }\end{array}$ & $\begin{array}{l}\text { Protéger un marché } \\
\text { Éliminer la concurrence déloyale } \\
\text { Convaincre les premiers acheteurs } \\
\text { Assainir un marché } \\
\text { Garantir une qualité minimale }\end{array}$ & 0,70 & \multirow{3}{*}{0,79} \\
\hline Corporate & $\begin{array}{l}\text { Travaux } \\
\text { normatifs } \\
\text { Mise en } \\
\text { conformité }\end{array}$ & $\begin{array}{l}\text { Accélérer la diffusion de sa } \\
\text { technologie } \\
\text { Gagner une avance } \\
\text { Retarder un concurrent } \\
\text { Être premier conforme } \\
\text { Être seul conforme }\end{array}$ & 0,63 & \\
\hline Business & $\begin{array}{l}\text { Pouvoir } \\
\text { d'entreprise }\end{array}$ & $\begin{array}{l}\text { Produire un volume plus important } \\
\text { Réduire la variété } \\
\text { Mieux gérer les stocks } \\
\text { Acheter moins cher } \\
\text { S'adresser à un marché plus large } \\
\text { Exporter dans différents pays } \\
\text { Différencier } \\
\text { Offrir un plus } \\
\text { Garantir la qualité des composantes }\end{array}$ & 0,68 & \\
\hline
\end{tabular}

Cette échelle permet d'établir une typologie selon les perceptions des normes par les responsables normalisation. Quatre types sont distingués: le premier, celui des « convaincus », affirme fortement les propriétés stratégiques des normes aux niveaux interorganisationnel, corporate et business, il représente $34 \%$ de l'échantillon. Le second type perçoit clairement l'effet développement au niveau interorganisationnel (l'effet protection n'est pas validé), les effets concurrentiels au niveau corporate et l'effet différenciation au niveau business. Il est composé de $38 \%$ de l'échantillon. Le troisième type affirme clairement l'effet développement au niveau interorganisationnel, mais invalide l'effet protection. Ce type considère que les normes peuvent permettre de gagner une avance sur les concurrents mais rejette l'idée que cet avantage puisse être agressif et ralentir les concurrents. Il ne considère pas les normes comme un instrument au niveau des produitsmarchés. Il représente $26 \%$ de l'échan- 
tillon. Enfin, le quatrième type n'est constitué que de $2 \%$ de l'échantillon. Il s'agit de dubitatifs, qui ne se prononcent pas sur les effets des normes sauf sur l'effet développement de marché, au travers de l'item « assainissement ». Cet item est isolé et traduit simplement le rôle de régulateur associé aux normes.

Lorsque l'on interroge les responsables marketing, sur la même échelle, ils sont davantage dubitatifs et dans le meilleur des cas, attribuent les propriétés stratégiques des normes au niveau business. Le groupe des « convaincus » représente $28 \%$ de l'échantillon, un groupe plus modéré représente $36 \%$ de l'échantillon, $31 \%$ nient les propriétés stratégiques des normes et $5 \%$ de l'échantillon ne se prononcent pas. Cette analyse montre une absence de cohérence entre les responsables des normes et du marketing, dont nous vérifions par ailleurs qu'ils ne se coordonnent pas pour définir des positions normatives communes au sein des travaux normatifs. Elle fait apparaître un groupe de convaincus qui rejoint le groupe proactif des responsables des normes, mais les convictions portent davantage sur le niveau business. Il semble donc que la position normative proactive n'exige pas une excellente cohérence entre responsable des normes et responsable marketing.

\section{La mesure du comportement vis-à-vis des normes}

La recherche permet de mettre au point une typologie des comportements vis-à-vis des normes sur un axe liant l'attentisme à la proactivité. Au cours des travaux normatifs, trois formes de comportements signalent une attitude proactive: la proposition de projets de norme, l'obtention de la pré- sidence ou du secrétariat des comités techniques, et le déclenchement de la procédure d'information. Par rapport à la mise en conformité, les entreprises peuvent anticiper la mise en conformité ou attendre qu'elle soit imposée par le marché. Aux deux points opposés de l'axe d'interprétation se situent les « proactifs » qui participent activement aux travaux et anticipent la mise en conformité aux normes, ils représentent $38 \%$ de l'échantillon. Le type des « opportunistes » qui participent occasionnellement aux travaux normatifs représente $16 \%$ de l'échantillon, les «suiveurs » sont rarement présents et ne prennent pas d'initiative dans les travaux, et les « réactifs », absents des travaux normatifs, ne s'intéressent aux normes qu'une fois instituées.

\section{Des modes de gestion liées aux caractéristiques des entreprises - test des hypothèses}

Les deux hypothèses principales sont vérifiées: La perception des propriétés stratégiques des normes et le comportement visà-vis des normes sont liées aux caractéristiques de l'entreprise et de son mode d'organisation des normes. Un mode proactif est identifié qui se traduit par la perception des propriétés stratégiques des normes et par un comportement proactif lors des travaux normatifs et dans la mise en conformité. Les entreprises qui mettent en œuvre cette stratégie proactive sont de grande taille, se positionnent en tant que leader, adoptent un profil innovateur dans le domaine technologique et ont un service normalisation dans l'entreprise, ce service étant le plus souvent directement lié à la direction générale. 


\section{IV. - DISCUSSION : LA NORME COMME INSTRUMENT DE STRATÉGIE COLLECTIVE}

Plusieurs éléments peuvent être discutés. En premier lieu, l'adoption des modèles de Porter peut être critiquée en ce que son approche est trop spécifiquement sectorielle. Nous devons considérer toutefois que l'approche de l'avantage concurrentiel en position sur le marché, a permis de plus facilement dissocier le niveau business ou niveau corporate, ce qui aurait été plus délicat avec le modèle fondé sur les ressources. En second lieu, le montage méthodologique prévoyant d'interroger le responsable des normes et le responsable de marketing se prête à un regard critique, car finalement, la perception de la part du responsable marketing ne s'est pas avérée nécessaire pour que les comportements proactifs soient réalisés. Un argument néanmoins favorable à l'audition des responsables marketing est précisément de vérifier que les normes peuvent être effectivement perçues comme variables d'intervention à certains niveaux (ici produitsmarchés), sans l'être à d'autres (interorganisationnel et corporate). Cela confirme que le modèle des stratégies collectives correspond effectivement à une stratégie spécifique.

Nous souhaitons également souligner qu'il serait illusoire d'associer systématiquement la participation aux travaux normatifs avec une stratégie proactive et la mise en conformité avec une stratégie réactive Enfin, Nous désirons montrer que les composantes concurrentielles et coopératives ne sont pas nécessairement contradictoires et incompatibles.

\section{Les dimensions réactive et proactive}

La typologie fait clairement apparaitre que les entreprises qui adoptent les normes comme modalité de stratégies collectives participent activement aux travaux normatifs, et l'implication remarquable, dans les travaux normatifs, des entreprises leaders et innovantes dans les secteurs technologiques est un élément confirmatif des enjeux spécifiques associés à la définition des normes pour promouvoir les technologies nouvelles. L'analyse des motivations fait apparaître les motifs liés aux capacités de diffusion des normes qui permettent de gagner une avance concurrentielle: les entreprises ayant un profil technicien affirment dans leur majorité $(63 \%)$ que les normes permettent de ralentir la diffusion d'une technologie concurrente, alors que les entreprises au profil commercial ne sont que $45 \%$ à percevoir cette propriété. Mais la participation aux travaux normatifs est tout d'abord défensive: il s'agit de surveiller l'environnement avant de tenter d'imprimer son influence. La nécessaire « veille normative » rend compte du pouvoir de structuration des normes sur un marché. L'émergence des normes est source d'incertitude car elle menace de bouleverser les règles du jeu concurrentiel et de favoriser voire de provoquer, ainsi, une rupture technologique. La participation aux travaux normatifs est donc un outil de réduction de l'incertitude liée à l'environnement: elle permet aux leaders d'imprimer leur influence sur les conditions concurrentielles, elle favorise la réactivité des entreprises qui cherchent une opportunité stratégique et permet aux suiveurs de préparer en douceur les mutations nécessaires. Si la 
participation aux travaux normatifs n'est pas nécessairement l'expression d'une intentionnalité proactive, le comportement de mise en conformité n'est pas non plus nécessairement réactif.

En effet, la conformité aux normes peut également signifier une intention proactive. La grande majorité des entreprises anticipe la date de promulgation des normes pour respecter les exigences normatives. L'anticipation de la date d'échéance de la norme peut exprimer la réactivité de l'entreprise (McKee et al., 1989) et signifier une gestion saine des modifications à apporter. Elle permet à certaines entreprises, motrices du marché par leur taille ou leur position, d'agir sur le marché de manière proactive: leur adoption des normes et la façon qu'elles choisissent pour satisfaire les exigences normatives sont susceptibles d'entraîner l'adhésion des entreprises fournisseurs et clientes. Elles contribuent ainsi à modifier les conditions de l'environnement. Dans les technologies de réseau, le comportement des premiers adopteurs provoque un effet boule de neige qui modifie la situation de choix de l'adopteur suivant en donnant du poids à la norme choisie (Arthur, 1987). D'autres marchés offrent une situation comparable: les grands acheteurs, les acheteurs institutionnels, les syndicats professionnels par leur choix d'une norme modifient largement les conditions de l'environnement, en favorisant les produits conformes et en évinçant littéralement les autres du marché. Le responsable normalisation de Gec Alsthom TGV mentionne l'exemple de l'adoption d'une nouvelle norme concernant la visserie qui avait provoqué une modification de structure importante et s'était soldée par un échec car les autres utilisateurs n'avaient pas suivi la nouvelle norme. Les vis conformes aux nouvelles normes ne se répandant pas sur le marché, elles n'offraient pas les conditions avantageuses liées aux économies d'échelles. Avant de se prononcer sur l'adoption ou non de tel élément de visserie qui présente des propriétés anticorrosives, un responsable de Merlin Gerin observe l'attitude de Renault. Certains gros acheteurs et les acheteurs institutionnels réalisent ainsi des arbitrages qui modifient manifestement les conditions de l'environnement.

Ainsi la participation aux travaux normatifs mais également la mise en conformité provoque des situations décisionnelles qui se positionnent sur un axe de réactivité-proactivité. La seconde dimension caractéristique est l'axe « concurrentiel-coopératif »

\section{Caractère concurrentiel ou coopératif}

Le concept de coopétition exprime bien la co-occurrence de compétition et de coopération. Les normes constituent une grille d'analyse pertinente pour en explorer les facettes, tant dans les travaux normatifs que dans la mise en conformité.

La coopération est le fondement même des stratégies collective. L'émergence ou la modification des normes joue un rôle souvent décisif dans les stratégies de rupture, en provoquant, à l'initiative et au profit d'une entreprise ou d'une groupe d'entreprises, une modification des règles du jeu concurrentiel. Or, la nature consensuelle du mode de définition des normes rend souvent nécessaire que ces stratégies soient coopératives: il s'agit de s'accorder pour défendre en groupe une position normative commune. 
Cette recherche d'une position commune des concurrents est rendue vitale dans le cas des marchés naissants. La phase de normalisation qui assainit le marché en garantissant une qualité minimale au client, est une phase indispensable pour convaincre les nouveaux clients et développer la demande initiale (Porter, 1985). L'entreprise réalise alors un arbitrage entre le développement de sa propre part de marché ou le développement du marché global, il s'agit de s'accorder sur les composantes à normaliser, et celles autorisant la différenciation des offres. Aussitôt que les conditions sont requises pour assurer la croissance du marché naissant, le jeu concurrentiel de développement de parts de marché peut reprendre place. L'enquête confirme la perception du rôle des normes pour le développement de la demande sur les marchés naissants.

De surcroît, l'utilité de la norme repose sur son appropriation par le marché. Cette propriété induit que l'avantage produit par une norme favorable ne constitue pas en soi un avantage unique et durable: la diffusion de la norme réduit les capacités de différenciation et la promulgation d'une norme favorable sert les intérêts de tout le marché. Diffuser son savoir-faire au moyen de la norme consiste à livrer son savoir-faire pour structurer la demande du marché. Le choix alternatif serait de protéger son savoir-faire au moyen de brevets. Ce choix conduirait à conserver un marché captif mais plus étroit. Le responsable de la normalisation France d'IBM confirme que le développement technologique est trop rapide pour se protéger, il faut diffuser pour orienter l'évolution technologique dans un sens favorable. Il faut aller grouper sur les marchés naissants. Ainsi les responsables normalisation de
Tefal et de Seb mentionnent leur attente de normes communes pour se lancer sur le marché de la domotique et les équipementiers citent également le cas de l'airbag pour expliquer ce comportement.

La dimension concurrentielle n'est pourtant jamais oubliée dans les stratégies collectives. Les entreprises qui se caractérisent par un mode actif et coopératif de comportement dans les travaux normatifs perçoivent avant tout que la normes peut favoriser l'entreprise par rapport à ses concurrents. Les dimensions concurrentielles et coopératives se trouvent donc inextricablement liées.

De fait, le croisement de l'ensemble des variables de l'étude, fait ressortir une cohérence très forte entre l'attente des propriétés de diffusion d'une norme favorable, la proposition de projets de normes, l'anticipation de la mise en conformité, et les attentes concurrentielles liées à la participation. Les entreprises convaincues du fait que les normes accélèrent la diffusion d'une nouvelle technologie sont les plus actives dans les travaux normatifs par les projets de normes qu'elles proposent. Leur participation est motivée par le souhait d'entériner les options prises par l'entreprise et de rentabiliser les efforts de recherche et développement. Cette attente proactive est liée à l'anticipation de la mise en conformité. L'entreprise propose des produits conformes aux exigences essentielles des normes avant même que les normes soient promulguées. Ce comportement est lié à l'attente des propriétés de diffusion des normes. Elle montre que les entreprises utilisent les capacités de diffusion des normes par la mise en conformité. Ces deux comportements s'intègrent dans les deux types de stratégies technologiques de l'innova- 
teur ou le premier à développer en masse le produit sur le marché.

L'entreprise participe aux travaux normatifs pour modeler des normes favorables à ses produits dans l'attente que les normes accéléreront la diffusion du produit. Elle peut aussi participer aux travaux pour être informée des attentes formulées et, sans avoir influencé le projet de norme, proposer un produit conforme à ces attentes, afin de bénéficier de l'effet de diffusion des normes. C'est ainsi que Philips, après avoir vainement lutté, tout au long des travaux normatifs, contre le seuil choisi de température de congélation, a été néanmoins le premier à proposer des produits conformes à la nouvelle norme. Le comportement est donc successivement voire simultanément concurrentiel et coopératif.

\section{CONCLUSION}

Le modèle des stratégies collectives fournit un cadre théorique permettant d'identifier des stratégies collectives proactives. De ce modèle, nous avons choisi de considérer deux dimensions essentielles que sont la proactivité et le caractère coopératif. Il s'avère que les normes offrent un terrain d'analyse particulièrement riche pour donner corps à ces stratégies.

Après avoir identifié, dans une phase qualitative que les normes pouvaient être perçues comme contrainte ou instruments aux différents niveaux, du marché, de l'entreprise ou des produits marchés, nous proposons une grille d'analyse associant les différentes dimensions perceptuelles et comportementales. En premier lieu, les propriétés d'organisation des conditions concurrentielles doivent être identifiées, notamment les effets structurants sur le marché. Cette analyse requiert l'étude des perceptions des propriétés stratégiques des normes. Elle nécessite également l'analyse des comportements: vis-à-vis des normes, la proactivité signifie agir sur la définition même des normes; dans l'entreprise, l'emploi des normes comme instrument de marché suppose une coordination entre le responsable de marché et le responsable normalisation; enfin, sur le marché, l'emploi des normes comme instrument stratégique suppose que les responsables de marché utilisent effectivement les normes.

L'enquête quantitative interrogeant le responsable des normes et le responsable marketing de 150 entreprises permet de proposer une échelle de mesure des perceptions des propriétés stratégiques des normes dont la fiabilité est vérifiée. Elle permet également d'offrir des perceptions et des comportements vis-à-vis des normes qui permettent de faire apparaître un mode collectif proactif de gestion des normes qui est illustratif des stratégies collectives. Ce mode proactif est le fait d'entreprises de grande taille, leaders sur leur marché, appartenant au secteur technologique et organisant la gestion des normes.

Un examen plus approfondi de ce mode proactif conduit à montrer que la proactivité se révèle au sein des travaux normatifs, mais peut également apparaître au moment de la mise en conformité. Il permet surtout de constater que les composantes individuelles et coopératives sont intimement liées. Les entreprises les plus dynamiques dans les travaux normatifs se caractérisent par une conception compétitive des normes. Ainsi, une procédure collective consensuelle ne signifie pas que les enjeux de pouvoir soient évincés. Faut-il s'en étonner? 


\section{Bibliographie}

Aldrich H., Organizations and environments, Englewood Cliffs, N. J., Prentice Hall, 1979. Pennings J., "Strategically interdependent organizations", Handbook of organizational design, vol. 1, Nystrom P.C. \& Starbuck W.H. (Eds), Oxford University Press, New York, 198, p. 433-4351.

Ackoff R., Redesigning the future, Wiley \& Sons, New York, 1974.

Aldrich H., Organizations and environments, Englewood cliffs, N. J., Prentice-Hall, 1979.

Andrews K., The concept of corporate strategy, Homewood, Dow-Jones-Irwin, 1971.

Ansoff I., Corporate strategy: An analytical approach to business policy for growth and expansion, New York, McGraw-Hill, 1965.

Arthur B., Competing Technologies: An Overview, Technical paper, Center for Economic Policy Research, July, Stanford University, Califormia, 1987.

Arthur B., "Competing technologies, increasing returns, and lock-in by historical events", The Economic Journal, The Quarterly Journal of the Royal Economic Society, 394, vol. 99, 1989, p. 116-132.

Astley W., Fombrun C., "Collective strategy: Social ecology of organizational environments", Academy of Management Review, vol. 8, 1983, p. 576-587.

Bourgeois L., "Strategy and environment: A conceptual integration", Academy of Management Review, 5, 1980, p. 25-40.

Brandenburger A., Nalebuff B., Co-opetition, éd. Village Mondial, 1996.

Carney M., "The strategy and structure of collective action", Organizational Studies, 8, p. 341-362.

Child J., "Organizational structure, environment and performance: The role of strategic choice", Sociology, 6, 1972, p. 1-22.

Cowan R., Foray D., «Économie de la codification et de la diffusion des connaissances », L'économie de l'Information: les enseignements des théories économiques, ed. Petit P., Éditions de la Découverte, Paris, 1998, p. 253-329.

Cyert R., March J., A behavioral theory of the firm, Wiley, New York, 1963.

David P., "Some New Standards for the Economics of Standardization in the Information Age", Economic Policy and Technological Performance, eds. Dasgupta P., Stoneman P. and P., Cambridge University Press, Cambridge, 1987, p. 206-239.

David P., Greenstein S., "The Economic of Compatibility Standard: An Introduction to Recent Research", Economics of Innovation and New Technology, vol. 1, 1990, p. 1-12.

Day G., Wensley R., "Assessing competitive advantage: a framework for diagnosing competitive superiority", Journal of Marketing, vol. 52, n 2, 1988, p. 1-20.

Di Maggio P., Powell W., "The iron cage revisited: institutional isomorphism and collective rationality in organizational fields", American Sociological Review, vol. 48, 1983, p. 147-160. Emery F., Trist E., "The causal texture of organizational environments", Human Relations, 18, 1965, p. 2-32.

Emshoff J., Freeman R., "Stakeholder management: a case study of the U.S. Brewers Association and the container issue", Applications of Management Science, 1, 1981, p. 57-90. 
Foray D., «Exploitation des externalités de réseau versus évolution des normes: les formes d'organisation face au dilemme de l'efficacité dans le domaine des technologies de réseau », Revue d'économie industrielle, $\mathrm{n}^{\circ}$ 51, $1^{\mathrm{er}}$ trimestre 1990, p. 113-139.

Foray D., «Standard de référence, coûts de transaction et économie de la qualité : un cadre d'analyse », Économie rurale, 217, septembre-octobre 1993, p. 33-41.

Hannan M., Freeman J., "The population ecology of organisations", American Journal of Sociology, 82, 1997, p. 929-964.

Hofer C., Schendel D., Strategy formulation: Analytical concepts, West publishing New York, 1978.

Katz M., Shapiro C., "Network Externalities Competition and Compatibility", American Economic Review, vol. 75, n 3, 1985, pp. 424-440.

Le Roy F., «Rivaliser et coopérer avec ses concurrents: le cas des stratégies collectives "agglomérées" », Revue française de gestion, vol. 29, n 143, mars-avril 2003, p. 145-157.

Lambin M., Day G., "Evolutionary processes in competitive markets: Beyond the product life cycle", Journal of Marketing, vol. 53, July 1989, p. 4-20.

Mason R., Mitrof I., Challenging strategic planning asumptions: theory, cases and techniques, Wiley, New York, 1981.

McKee D., Varadarajan P., Pride W., "Strategic adpatatbility and firm performance: a market contingent perspective", Journal of Marketing, vol. 53, n 3, July 1989, p. 21-35.

Michael D., On learning to plan and planning to learn, Jossey-Bass, San Francisco, 1973.

Miles R., Snow C., Organizational stratgey structure and process, Mc Graw Hill, New York, 1978.

Mione A., Les normes, instrument de la stratégie marketing de l'entreprise, Thèse de doctorat en Sciences de gestion, université Montpellier II, 1994.

Mione A., Fenneteau H., « Normes, certification ISO 9000 et compétitivité des entreprises », Management de la compétitivité et de l'emploi, Pérez R., Brabet J., S. Yami (ed.), L'Harmattan, Paris, 2004.

Pennings J., "Strategically interdependent organizations", Handbook of organizational design, vol. 1, P.C. Nystrom \& W.H. Dstarbuck (Eds), Oxford University Press, New York, 1981, pp. 433-435.

Pfeffer J., Salancik G., The external control of organisations, New York, Harper \& Row, 1978.

Porter M., Competitive advantage; creating ans sustaining superior performance, The Free Press, McMillan Inc., New York, 1985.

Poissonnier H., « Nature et dynamique des stratégies agglomérées développées par les fournisseurs dans la filière textile-habillement-distribution », atelier AIMS Stratégies collectives, Montpellier.

Porter M., Millar V., "How information gives you competitive advantage", Harvard Business Review, $\mathrm{n}^{\circ}$ 4, July-August 1985, p. 149-160.

Schon D., Beyond the stable, Basic Books, New York, 1971. 
122 Revue française de gestion $-\mathrm{N}^{\circ} 167 / 2006$

Trist E., "Referent organizations and the development of interorganizational domain", Distinguished Lecture, Organization and Management theory Division, $39^{\text {th }}$ Annual Convention of the Academy of Management, Atlanta, Georgia, 1979.

Vickers G., The art of jugement, Chapman and Hall, London, 1965.

Yami S., « Petite entreprise et stratégie collective de filières », Revue française de gestion, vol. 29, n 144, mai-juin 2003, p. 165-179. 